\title{
Magnetic moment of the $2083 \mathrm{keV}$ level of ${ }^{140} \mathrm{Ce}$
}

\author{
Y. Ohkubo, ${ }^{1}$ A. Taniguchi, ${ }^{1}$ Q. Xu, ${ }^{1}$ M. Tanigaki, ${ }^{1}$ N. Shimizu, ${ }^{2}$ and T. Otsuka ${ }^{2,3}$ \\ ${ }^{1}$ Research Reactor Institute, Kyoto University, Kumatori-cho, Sennan-gun, Osaka 590-0494, Japan \\ ${ }^{2}$ Center for Nuclear Study, University of Tokyo, Hongo, Bunkyo-ku, Tokyo 113-0033, Japan \\ ${ }^{3}$ Department of Physics, University of Tokyo, Hongo, Bunkyo-ku, Tokyo 113-0033, Japan \\ (Received 6 November 2012; revised manuscript received 17 February 2013; published 15 April 2013)
}

\begin{abstract}
For the magnetic moment of the $2083 \mathrm{keV}$ level of ${ }^{140} \mathrm{Ce}$, there are four published data, all obtained by applying an external magnetic field of less than $5 \mathrm{~T}$ to a liquid sample containing ${ }^{140} \mathrm{La}$ using the time-differential perturbed angular correlation (TDPAC) technique. Although these four values are consistent within two times their uncertainties $(2 \sigma)$, the range of values in $2 \sigma$ extends from $\mu=+3.0$ to +5.2 (in units of nuclear magneton, $\mu_{\mathrm{N}}$ ). This time, the TDPAC technique was successfully applied to the $2083 \mathrm{keV}$ level of ${ }^{140} \mathrm{Ce}$ implanted in an Fe foil. The magnetic moment of this level was determined to be $\mu=+4.00(20) \mu_{\mathrm{N}}$, employing the known hyperfine field at ${ }^{141} \mathrm{Ce}$ in $\mathrm{Fe},-41(2) \mathrm{T}$, which agrees very well with one of the values, $\mu=+4.06(15) \mu_{\mathrm{N}}$. The present value is compared with two shell-model calculations.
\end{abstract}

DOI: 10.1103/PhysRevC.87.044324 PACS number(s): 21.10.Ky, 23.20.En, 27.60.+j, 29.38.-c

\section{INTRODUCTION}

Because its neutron number 82 is magic, ${ }^{140} \mathrm{Ce}$ is an important nuclide for nuclear-structure study. Interplay between single-particle and collective degrees of freedom can be studied in low-lying levels in ${ }^{140} \mathrm{Ce}$. From an electron-scattering experiment and a calculation based on the quasiparticle phonon model, its $2083 \mathrm{keV}$ level having a nuclear spin of $I=4$ was interpreted as a two-quasiparticle state, although with a substantial collective strength [1]. Moreover, this level is a useful nuclear probe for materials science, concerning the behavior of the $4 f$ electron in particular [2]. The magnetic moment of the $2083 \mathrm{keV}$ level of ${ }^{140} \mathrm{Ce}$ is one of key physical quantities reflecting the character of the nuclear wave function of this level. In Ref. [3] are listed four published values for the magnetic moment of the $2083 \mathrm{keV}$ level of ${ }^{140} \mathrm{Ce}$ : (in units of nuclear magneton, $\left.\mu_{\mathrm{N}}\right) \mu=+4.06(15)$ [4], +3.8(4) [5], $+4.44(16)$ [6], and $+4.6(3)$ [7]. Although these four values are consistent within two times their uncertainties $(2 \sigma)$, the range of the values in $2 \sigma$ extends from $\mu=+3.0$ to $+5.2 \mu_{\mathrm{N}}$, as shown in Fig. 1. It is desirable to improve this situation. These values were all obtained by applying an external magnetic field of less than $5 \mathrm{~T}$ to a liquid sample containing ${ }^{140} \mathrm{La}$ using the time-differential perturbed angular correlation (TDPAC) technique. Since the half-life of the $2083 \mathrm{keV}$ level of ${ }^{140} \mathrm{Ce}$ is $3.4 \mathrm{~ns}$, it is difficult to observe many oscillations in the TDPAC spectrum with an external field of less than about $5 \mathrm{~T}$.

In this paper we report our TDPAC measurement of the magnetic moment of the $2083 \mathrm{keV}$ level in ${ }^{140} \mathrm{Ce}$ diluted in an $\mathrm{Fe}$ foil, using the known value of the magnetic hyperfine field $B_{\mathrm{hf}}$ at ${ }^{141} \mathrm{Ce}$ in $\mathrm{Fe}$ at a few $\mathrm{mK},-41(2) \mathrm{T}$ [8], and compare the obtained magnetic moment with the results of shell-model calculations utilizing two residual interactions: one is the $Q$-box interaction of the $G$ matrix derived from CD-Bonn nucleon-nucleon interaction (CD-Bonn $+G$ matrix) used in Ref. [9] and the other consists of monopole pairing, quadrupole pairing, and quadrupole-quadrupole interactions $(\mathrm{P}+\mathrm{QQ})$ used in Ref. [10].

\section{EXPERIMENTAL PROCEDURES}

\section{A. Sample preparation}

Lanthanum does not form a solid solution with iron. The ion implantation was performed at the solid-state physics beam course of the online isotope separator at the Research Reactor Institute, Kyoto University (KUR-ISOL) [11]. ${ }^{140} \mathrm{Cs}$ producing ${ }^{140} \mathrm{La}$ were obtained by the fission reaction of a $50 \mathrm{mg}{ }^{235} \mathrm{U}$ target with thermal neutrons of $3 \times 10^{12} \mathrm{~cm}^{-2} \mathrm{~s}^{-1}$ and were carried to the surface ionizer by a $\mathrm{PbI}_{2}$ aerosol in a mixed-gas jet of $\mathrm{He}$ and $\mathrm{N}_{2}$. The ionized fission products, mainly alkalimetal elements, were accelerated to $30 \mathrm{keV}$ for mass analysis. Mass-separated ${ }^{140} \mathrm{Cs}$ ions were further accelerated to $100 \mathrm{keV}$ by the postaccelerator to be implanted at room temperature in a $10 \mathrm{~mm} \times 10 \mathrm{~mm} \times 0.1 \mathrm{~mm}$ Fe foil of $99.995 \%$ purity, which had been annealed in $\mathrm{H}_{2}$ atmosphere at $700^{\circ} \mathrm{C}$ for $2 \mathrm{~h}$ and then polished. The diameter of the ${ }^{140} \mathrm{Cs}$ beam size was about $5 \mathrm{~mm}$. The implantation depth was estimated to be $20 \pm 10 \mathrm{~nm}$. The parent nuclei of ${ }^{140} \mathrm{Ce},{ }^{140} \mathrm{La}$, were produced in the radioactive equilibrium of ${ }^{140} \mathrm{Ba}^{140} \mathrm{La}$, followed by the $\beta$ decay of ${ }^{140} \mathrm{Cs}$. A relevant part of the decay scheme of the $A=140$ mass chain is shown in Fig. 2 .

\section{B. Time-differential perturbed angular correlation measurements}

The time dependence $N(\theta, t)$ of the coincidence counts of the $329-487 \mathrm{keV}$ cascade $\gamma$ rays for the Fe foil containing ${ }^{140} \mathrm{La}$ was taken at room temperature with a measurement system consisting of standard fast-slow electronic modules and four or three 1.5 inch $\phi \times 1.5$ inch $\mathrm{BaF}_{2}$ scintillation detectors. Here, $\theta$ and $t$ denote the angle and the time interval, respectively, between the cascade $\gamma$ rays.

Using four detectors, the directional anisotropy $A_{22} G_{22}(t)$ was obtained according to

$$
A_{22} G_{22}(t)=2 \frac{N\left(180^{\circ}, t\right)-N\left(90^{\circ}, t\right)}{N\left(180^{\circ}, t\right)+2 N\left(90^{\circ}, t\right)} .
$$




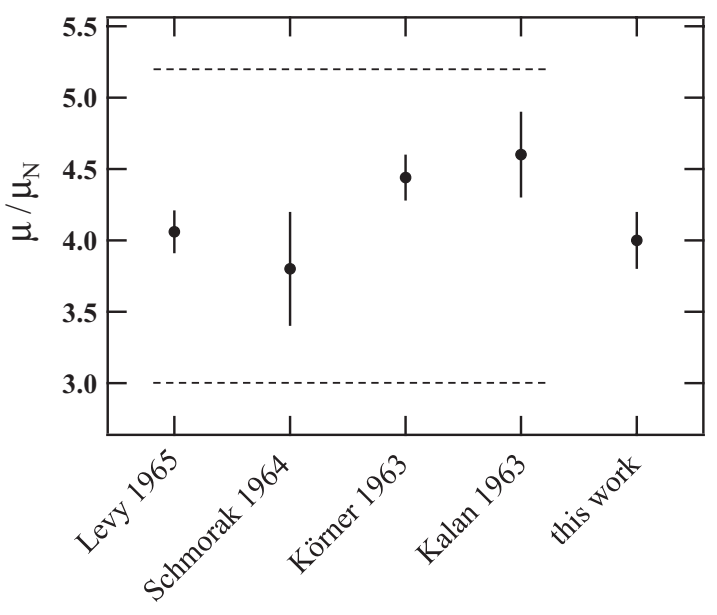

FIG. 1. Reported values of the magnetic moment, $\mu$, of the $2083 \mathrm{keV}$ level in ${ }^{140} \mathrm{Ce}$ in units of the nuclear magneton $\left(\mu_{\mathrm{N}}\right)$ including the present value (Levy 1965 [4], Schmorak 1964 [5], Körner 1963 [6], and Kalan 1963 [7]). The dashed lines represent the upper and lower of the four reported values with $2 \sigma$.

The $10 \mathrm{~mm} \times 10 \mathrm{~mm}$ plane of the Fe foil was parallel to the detector plane. The distance between the source and each detector was $3.0 \mathrm{~cm}$. With four detectors, a slight asymmetry in the detectors is eliminated in the operation of Eq. (1). ${ }^{1}$

Using three detectors with an external magnetic field from a cage-type magnet of $0.3 \mathrm{~T}$ [12] applied perpendicularly to the detector plane, the directional anisotropy $R(t)$ was obtained according to

$$
R(t)=\frac{4}{3} \frac{N\left(-135^{\circ}, t\right)-N\left(+135^{\circ}, t\right)}{N\left(-135^{\circ}, t\right)+N\left(+135^{\circ}, t\right)}
$$

in order to determine the sign of the magnetic moment. The $10 \mathrm{~mm} \times 10 \mathrm{~mm}$ plane of the $\mathrm{Fe}$ foil was perpendicular to the detector plane so that the demagnetization field was small; on the order of $0.01 \mathrm{~T}$ [13]. The distance between the source and each detector was $4.0 \mathrm{~cm}$. We performed two sets of the TDPAC measurements by inverting the direction of the external magnetic field, leaving the detector arrangements untouched, and derived the following two quantities by subtracting or adding the two sets of $R(t)$ data:

$$
R_{\mathrm{S}}(t)=\frac{R_{+}(t) / \sigma_{+}(t)^{2}-R_{-}(t) / \sigma_{-}(t)^{2}}{1 / \sigma_{+}(t)^{2}+1 / \sigma_{-}(t)^{2}},
$$

and

$$
R_{\mathrm{A}}(t)=\frac{R_{+}(t) / \sigma_{+}(t)^{2}+R_{-}(t) / \sigma_{-}(t)^{2}}{1 / \sigma_{+}(t)^{2}+1 / \sigma_{-}(t)^{2}}
$$

\footnotetext{
${ }^{1}$ Let $\varepsilon_{i}(j)$ denote the counting efficiency of detector $i$ for the $j$ th $\gamma$ ray, $i$ being 1, 2, 3, 4 and $j$ being $1(329 \mathrm{keV})$ and $2(487 \mathrm{keV})$. In the present four-detector setup where the anode signals of detectors 1 and 2 are connected to the start input of a time-to-amplitude converter (TAC) module and those of detectors 3 and 4 to the stop input, both $N\left(90^{\circ}, t\right)$ and $N\left(180^{\circ}, t\right)$ are proportional to the same quantity, $\left[\Pi_{i, j} \varepsilon_{i}(j)\right]^{1 / 4}$. In the operation of Eq. (1), these quantities in the numerator and denominator cancel each other.
}

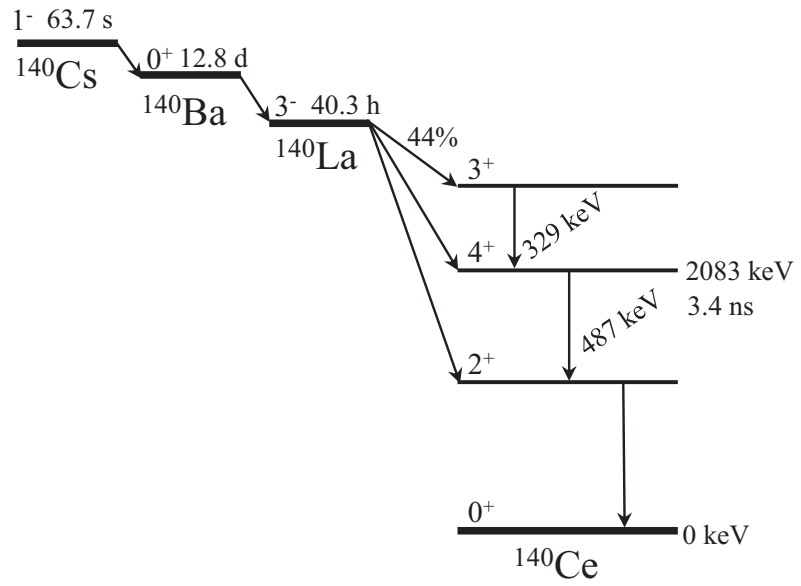

FIG. 2. Simplified decay scheme of relevant $A=140$ mass chain.

Here the subscripts + and - refer to the normal and reversed directions of the magnetic field, respectively, i.e., $R_{+}(t)$ equals $R(t)$ defined in Eq. (2) and $R_{-}(t)$ equals $-R(t)$, and $\sigma(t)$ represents the statistical uncertainty attached to the corresponding $R(t)$. With $R_{\mathrm{S}}(t)$, we can extract a pattern due to magnetic interactions. Using three detectors, a slight asymmetry in the two detectors at $\pm 135^{\circ}$ is cancelled in the $R_{\mathrm{A}}$ operation, but in the $R_{\mathrm{S}}$ operation it adds a constant term in the TDPAC spectrum.

The coefficient $A_{22}$ depends only on the nuclear transitions and its value for ${ }^{140} \mathrm{Ce}$ is reported to be -0.13 [14]. The perturbation factor $G_{22}(t)$ for an ensemble of randomly oriented microcrystals is a function of the Larmor frequency $\omega_{\mathrm{L}}$ for a unique static magnetic interaction. The quantity $\omega_{\mathrm{L}}$ is defined as usual: $\omega_{\mathrm{L}}=-\mu B /(I \hbar)$, where $B$ is the magnetic field at the nucleus. In the $180^{\circ}-90^{\circ}$ angular correlation case with no external magnetic field,

$$
A_{22} G_{22}(t)=A_{22}\left[1+2 \cos \left(\omega_{L} t\right)+2 \cos \left(2 \omega_{L} t\right)\right] / 5,
$$

having the constant term (which we call the baseline in the text) of $A_{22} / 5$.

In the $\pm 135^{\circ}$ angular correlation case with an external magnetic field,

$$
R(t)=A_{22} \sin \left(2 \omega_{L} t\right),
$$

having zero baseline.

\section{RESULTS AND COMPARISON WITH PREVIOUS RESULTS AND WITH SHELL-MODEL CALCULATIONS}

The obtained $A_{22} G_{22}(t)$ time spectrum is shown in Fig. 3 and the $R_{\mathrm{S}}(t)$ and $R_{\mathrm{A}}(t)$ time spectra are shown in Figs. 4(a) and 4(b), respectively, all of which have been corrected for the finite solid angle. Figures 3 and 4(a) display clear oscillation patterns. As should be, essentially no oscillation pattern is seen in Fig. 4(b) and $R_{\mathrm{A}}$ is nearly zero, except in the time range between 0 and about $2 \mathrm{~ns}$. We consider the oscillation in this time range due to the large prompt peaks in the $N(\theta, t)$ time spectra and therefore treat the other spectra in this time range as useless for the analysis. As described below Eq. (4), a slight 


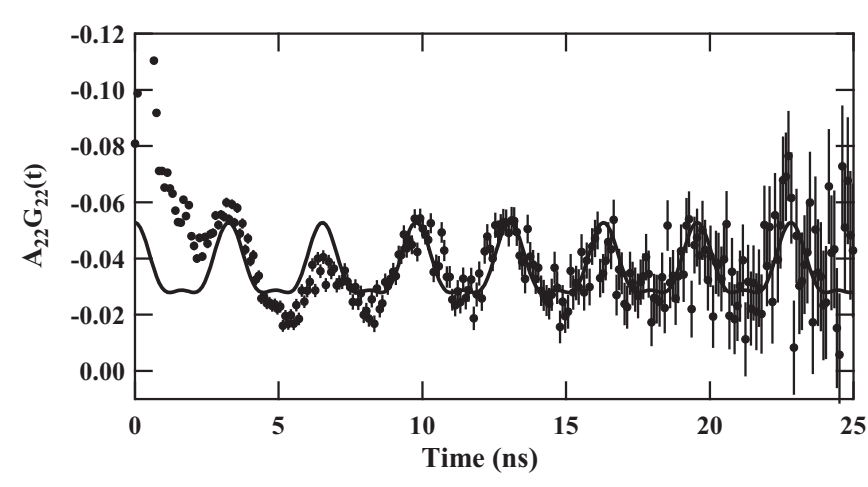

FIG. 3. TDPAC spectrum, $A_{22} G_{22}(t)$, of ${ }^{140} \mathrm{Ce}$ in $\mathrm{Fe}$ at room temperature using four $\mathrm{BaF}_{2}$ scintillation detectors without external magnetic field. The solid curve represents the fit of Eq. (5) in the text taking into account the finite time resolution of the TDPAC measurement system.

asymmetry in the two detectors at $\pm 135^{\circ}$ is responsible for the nonzero baseline of the TDPAC spectrum in Fig. 4(a). The fact that there is an oscillation pattern in Fig. 4(a) signifies that the oscillation patterns seen in Figs. 3 and 4(a) are due to the magnetic hyperfine interaction. The observation that the apparent oscillation period in Fig. 3 is twice as large as that in Fig. 4(a) implies that we can see mainly the oscillation due to the second term in Eq. (5), which is in turn due to the finite time resolution of the present detection system, 1 ns, about 10 times larger than the time bin $\Delta t$ of the time spectra. Residual lattice damage caused by ion implantation would lead to a distribution of hyperfine fields at the probe sites, which would cause a frequency distribution, resulting in a damping of the oscillation pattern of the TDPAC spectrum. Since the oscillations in Figs. 3 and 4(a) seem not to fade out in the present time range, the interaction involved is considered to be a unique static magnetic hyperfine interaction on ${ }^{140} \mathrm{Ce}$

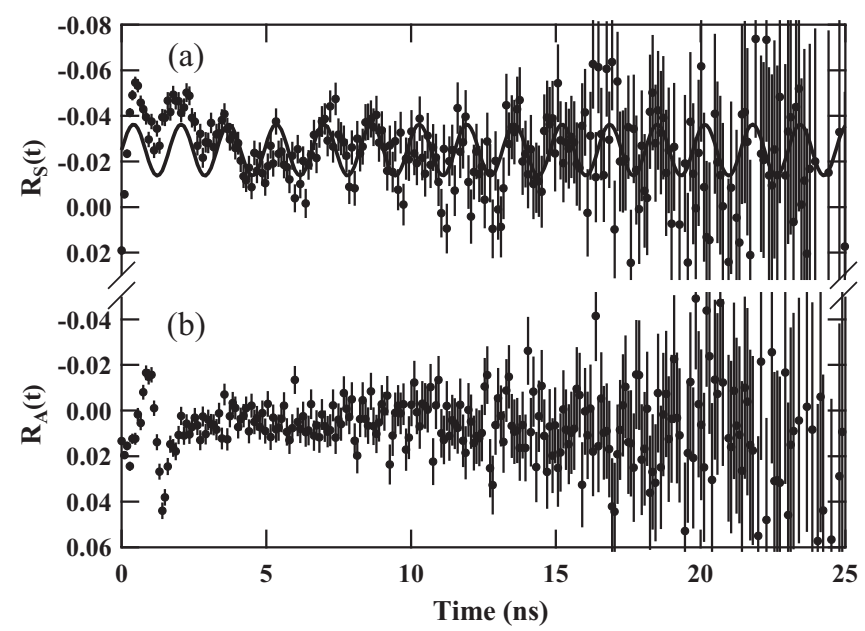

FIG. 4. TDPAC spectra, (a) $R_{\mathrm{S}}(t)$ and (b) $R_{\mathrm{A}}(t)$, of ${ }^{140} \mathrm{Ce}$ in $\mathrm{Fe}$ at room temperature using three $\mathrm{BaF}_{2}$ scintillation detectors with an external magnetic field of $0.3 \mathrm{~T}$. The curve in panel (a) was the result of the fit using Eq. (6) in the text with a constant term and the effective $A_{22}$ value determined in the fit in Fig. 3 . occupying the Fe substitutional site with no lattice defects nearby.

In addition to this component, other components contribute to the TDPAC spectrum in the time range between 0 and about $8 \mathrm{~ns}$, as seen in Fig. 3 in particular. Noting that the baseline of the TDPAC spectrum in Fig. 3 is nearly equal to $A_{22} / 5(=-0.026)$, which is the first term of Eq. (5), these components could be due to static perturbations and we thus tentatively consider that these components arise from those ${ }^{140} \mathrm{Ce}$ at interstitial sites or at the $\mathrm{Fe}$ sites having lattice defects nearby, feeling a variety of hyperfine magnetic fields and electric field gradients such that the oscillations arising from this variety of fields interfere among themselves and get dumped quickly in the $A_{22} G_{22}(t)$ time spectrum.

In order to determine the magnetic moment of the $2083 \mathrm{keV}$ level of ${ }^{140} \mathrm{Ce}$, we consider only the oscillation part of the TDPAC spectrum in Fig. 3 and assume that the corresponding ${ }^{140} \mathrm{Ce}$ occupy the $\mathrm{Fe}$ substitutional sites with no lattice defects nearby. The solid line in Fig. 3 represents the fit of the expression using Eq. (5) corrected for the finite time resolution of the detection system to the data in the time range between 2.3 and 24.8 ns. The amplitude of the oscillation, i.e., the effective $A_{22}$ value, is -0.042 , which is much smaller than the reported $A_{22}$ value of -0.13 , indicating that only about $30 \%$ of the ${ }^{140} \mathrm{Ce}$ implanted in $\mathrm{Fe}$ feel the unique static magnetic hyperfine interaction. This value agrees with the fraction of Ce considered to occupy the substitutional Fe site, 28.9(5)\%,

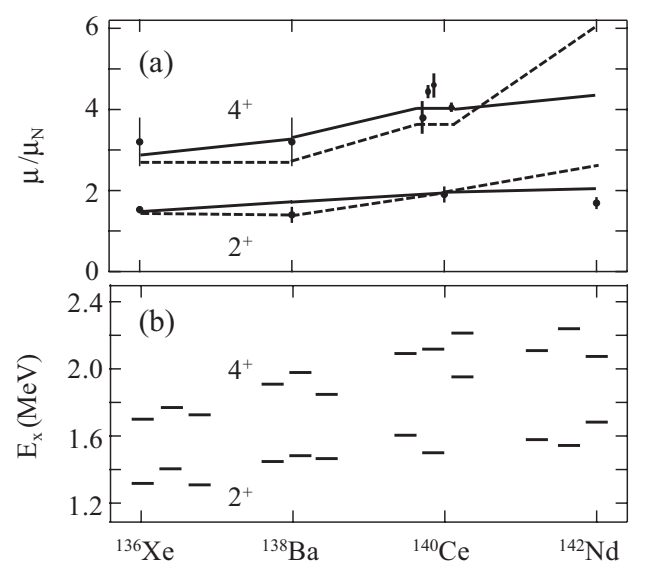

FIG. 5. (a) Magnetic moments (in $\mu_{\mathrm{N}}$ ) of the first $2^{+}$and $4^{+}$ levels of ${ }^{136} \mathrm{Xe},{ }^{138} \mathrm{Ba},{ }^{140} \mathrm{Ce}$, and ${ }^{142} \mathrm{Nd}$ (there is no experimental value available of the magnetic moment of the $4^{+}$level in ${ }^{142} \mathrm{Nd}$ ). The solid circles represent the experimental values taken form Ref. [3] except for the $4^{+}$level of ${ }^{140} \mathrm{Ce}$, for which from the left to the right are displayed the values from Refs. [5-7], and the average of the value in Ref. [4] and the present value. The solid lines for the $2^{+}$and $4^{+}$levels signify the shell-model calculation with the CD-Bonn $+G$ matrix (for brevity, SM/CD-Bonn), and the dashed lines signify the shell-model calculation with the $\mathrm{P}+\mathrm{QQ}$ interaction (SM/P $+\mathrm{QQ})$. (b) Excitation energies (in $\mathrm{MeV}$ ) of the first $2^{+}$and $4^{+}$levels of ${ }^{136} \mathrm{Xe},{ }^{138} \mathrm{Ba},{ }^{140} \mathrm{Ce}$, and ${ }^{142} \mathrm{Nd}$. For each nuclide, the left column denotes the experiment [3], the middle column denotes the SM/CDBonn calculation, and the right column denotes the SM/P + QQ calculation. In each column, the upper line stands for the $4^{+}$level and the lower for the $2^{+}$level. 
obtained in a nuclear orientation measurement on ${ }^{141} \mathrm{Ce}$ in $\mathrm{Fe}$ [15], although ${ }^{141} \mathrm{Ce}$ ions were directly implanted into an $\mathrm{Fe}$ foil, ${ }^{2}$ while in our case ${ }^{140} \mathrm{Cs}$ ions, the precursor of ${ }^{140} \mathrm{Ce}$, were implanted. The curve in Fig. 4(a) is the result of the fit using Eq. (6) with a constant term and the effective $A_{22}$ value determined in the fit in Fig. 3. Determining the sign of $\omega_{\mathrm{L}}$ to be positive from Fig. 4(a), the $\omega_{\mathrm{L}}$ value thus obtained from the $A_{22} G_{22}$ spectrum is $+1927(7) \mathrm{Mrad} / \mathrm{s}$ and that obtained from the $R_{\mathrm{S}}$ spectrum is $+1910(8) \mathrm{Mrad} / \mathrm{s}$. The observation that the $\omega_{\mathrm{L}}$ value obtained under the external magnetic field is smaller than that without it shows that the direction of the hyperfine magnetic field is opposite to that of the external magnetic field. From the two $\omega_{\mathrm{L}}$ values and the value of the external field, the hyperfine magnetic field may be estimated. However, it is $-34(21) \mathrm{T}$, which has a large uncertainty.

A much more reliable value of $B_{\mathrm{hf}}$ for ${ }^{141} \mathrm{Ce}$ in $\mathrm{Fe}$ is available: $-41(2) \mathrm{T}$ at a low temperature, which was obtained with the technique of nuclear magnetic resonance on oriented nuclei (NMR/ON) [8]. ${ }^{3}$ Assuming that $B_{\mathrm{hf}}$ scales with temperature according to the temperature dependence of the $\mathrm{Fe}$ magnetization [16], the value of $B_{\mathrm{hf}}$ at room temperature is about $2 \%$ reduced from that at a few $\mathrm{mK}{ }^{4}$ Using this value of $B_{\mathrm{hf}}$ and the $\omega_{\mathrm{L}}$ value of $+1927(7) \mathrm{Mrad} / \mathrm{s}$, the magnetic moment of the $2083 \mathrm{keV}$ level of ${ }^{140} \mathrm{Ce}$ was derived to be $+4.00(20) \mu_{\mathrm{N}}$. For the case of the $\omega_{\mathrm{L}}$ value

\footnotetext{
${ }^{2}$ As for the ionic state of ${ }^{141} \mathrm{Ce}$, it is concluded in Ref. [8] that ${ }^{141} \mathrm{Ce}$ in $\mathrm{Fe}$ is either in a diamagnetic $\mathrm{Ce}^{4+}$ state or in a state of mixed valence with a highly reduced $4 f$-shell contribution.

${ }^{3}$ In the NMR/ON experiment in Ref. [8], the sign of the magnetic hyperfine field, the value of $\mu(1+K) / I$, and the absolute value of $\mu B_{\text {hf }} / I$ were determined from the external magnetic field dependence of the resonance frequency. Here, $I, \mu$, and $K$ are, respectively, the nuclear spin, the nuclear magnetic moment, and the Knight shift. Assuming that $K$ was negligibly small and probably using the quadrupole interaction constant of $+1(4) \mathrm{MHz}$, the value of $B_{\mathrm{hf}}$ was derived to be $-41(2) \mathrm{T}$. We attempted to fit our data taking into account a quadrupole interaction in addition to the hyperfine magnetic interaction on a same ${ }^{140} \mathrm{Ce}$, but a better fit was not obtained than the case with no quadrupole interaction (addition of a small quadrupole interaction in the fit, which hardly changes the $\omega_{\mathrm{L}}$ value, damps the magnetic oscillation pattern with increasing time), implying that ${ }^{140} \mathrm{Ce}$ in $\mathrm{Fe}$ that feels a unique static magnetic hyperfine interaction is in a diamagnetic $\mathrm{Ce}^{4+}$ state.

${ }^{4}$ We note that there is an experimental result showing that the temperature dependence of a hyperfine magnetic field is larger than that of the saturation magnetization of Fe. While the ratio of the latter at $293 \mathrm{~K}$ to that at $4 \mathrm{~K}$ is $0.98151(6)$ [16], the ratio of the former for ${ }^{99} \mathrm{Tc}$ in $\mathrm{Fe}$ at $290 \mathrm{~K}$ to $10 \mathrm{~K}$ is $0.96862(19)$, described in Ref. [17].
}

of $+1910(8) \mathrm{Mrad} / \mathrm{s}$, the magnetic moment was derived to be $+3.99(20) \mu_{\mathrm{N}}$, taking into account the external magnetic field of $0.3 \mathrm{~T}$. The uncertainties in the parentheses composed of the statistical ones in the $\omega_{\mathrm{L}}$ values and the uncertainty in the $B_{\mathrm{hf}}$ value are due essentially to the latter uncertainty. The average becomes $+4.00(20) \mu_{\mathrm{N}}$. Figure 1 also shows the present result. Our value agrees well with the value obtained by Levy and Shirely in Ref. [4]: $+4.06(15) \mu_{\mathrm{N}}$. The average of these two is $+4.04(12) \mu_{\mathrm{N}}$ and is referred to hereafter as the LS-Oh value.

Shell-model calculations were performed on the first $2^{+}$and $4^{+}$levels of ${ }^{136} \mathrm{Xe},{ }^{138} \mathrm{Ba},{ }^{140} \mathrm{Ce}$, and ${ }^{142} \mathrm{Nd}(N=82$ isotones $)$, using two interactions, CD-Bonn $+G$ matrix [9] and pairing + quadrupole-quadrupole (P+QQ) [10]. The model space consisted of $s_{1 / 2}, d_{5 / 2}, d_{3 / 2}, g_{7 / 2}$, and $h_{11 / 2}$ proton orbits outside the closed ${ }^{132} \mathrm{Sn}$ core (the neutrons form a closed shell with $N=82$ ). Throughout these calculations, the same effective proton orbital and spin $g$ factors, $g_{l}$ and $g_{s}$, were used: $g_{l}=$ 1.0 and $g_{s}=0.7 \cdot g_{s}$ (free proton) $=3.91$, where the value of 0.7 is a standard spin-quenching factor. The results for the magnetic moments and excitation energies are summarized in Fig. 5, together with the corresponding experimental values taken from Ref. [3] and the LS-Oh value of $+4.04(12) \mu_{\mathrm{N}}$ for the $4^{+}$level of ${ }^{140} \mathrm{Ce}$. The agreement between each calculation and experiment is basically good. To be more precise, however, for the magnetic moment of the $2083 \mathrm{keV}$ level of ${ }^{140} \mathrm{Ce}$, the shell-model calculation with the CD-Bonn $+G$ matrix appears to be closer to the LS-Oh value than that with the P+QQ interaction. On the other hand, the situation is opposite for the $2^{+}$magnetic moment of ${ }^{138} \mathrm{Ba}$. Thus, the present experiment indicates that one needs further theoretical developments for a unified description with a sufficiently high precision.

\section{CONCLUSIONS}

We implanted ${ }^{140} \mathrm{Cs}$ into an Fe foil and successfully observed oscillation patterns due to a unique magnetic hyperfine interaction in the TDPAC spectra. Using the known value of $B_{\mathrm{hf}}$ at ${ }^{141} \mathrm{Ce}$ in $\mathrm{Fe}$, we derived the value of the magnetic moment of the $2083 \mathrm{keV}$ level in ${ }^{140} \mathrm{Ce}$ to be $+4.00(20) \mu_{\mathrm{N}}$, which is in excellent agreement with $+4.06(15) \mu_{\mathrm{N}}$ obtained by Levy and Shirley. The average of these two (the $\mathrm{LS}-\mathrm{OH}$ value) is $+4.04(12) \mu_{\mathrm{N}}$. Two shell-model calculations, one with the CD-Bonn $+G$ matrix and the other with the P+QQ interaction, reproduce fairly well the $\mathrm{LS}-\mathrm{OH}$ value, although the former calculation seems better. The present study thus provides a clear case that reliable experimental magneticmoment values are useful to evaluate various calculations.
[1] W. Kim, B. L. Miller, J. R. Calarco, L. S. Cardman, J. P. Connelly, S. A. Fayans, B. Frois, D. Goutte, J. H. Heisenberg, F. W. Hersman, V. Meot, T. E. Milliman, P. Mueller, C. N. Papanicolas, A. P. Platonov, V. Yu. Ponomarev, and J. E. Wise, Phys. Rev. C 45, 2290 (1992).

[2] A. W. Carbonari, J. Mestnik-Filho, R. N. Saxena, and M. V. Lalić, Phys. Rev. B 69, 144425 (2004).
[3] N. J. Stone, At. Data Nucl. Data Tables 90, 75 (2005); N. J. Stone, INDC(NDS)-0594 (2011).

[4] R. M. Levy and D. A. Shirley, Phys. Rev. 140, B811 (1965).

[5] M. Schmorak, H. Wilson, P. Gatti, and L. Grodzins, Phys. Rev. 134, B718 (1964).

[6] H. J. Körner, E. Gerdau, C. Günther, K. Auerbach, G. Mielken, G. Strube, and E. Bodenstedt, Z. Phys. 173, 203 (1963). 
[7] N. Kalan, S. Ofer, and B. Rosner, Phys. Lett. 3, 291 (1963).

[8] W. van Rijswijk, F. G. van den Berg, W. R. Joosten, and W. J. Huiskamp, Hyperfine Interact. 15/16, 325 (1983).

[9] B. A. Brown, N. J. Stone, J. R. Stone, I. S. Towner, and M. Hjorth-Jensen, Phys. Rev. C 71, 044317 (2005).

[10] N. Shimizu, T. Otsuka, T. Mizusaki, and M. Honma, Phys. Rev. Lett. 86, 1171 (2001).

[11] K. Okano, Y. Kawase, K. Kawade, H. Yamamoto, M. Hanada, T. Katoh, and I. Fujiwara, Nucl. Instrum. Methods Phys. Res. 186, 115 (1981); Y. Kawase, K. Okano, and F. Funakoshi, Nucl. Instrum. Methods Phys. Res., Sect. A 241, 305 (1985).
[12] M. Tanigaki, S. Izumi, H. Ouchi, A. Sasaki, Y. Miyashita, N. Sato, S. Hoshino, K. Shimada, T. Wakui, T. Shinozuka, and Y. Ohkubo, Phys. Rev. C 80, 034304 (2009).

[13] S. Chikazumi, Physics of Magnetism (Wiley, New York, 1964).

[14] B. Klemme and H. Miemczyk, J. Phys. Soc. Jpn. 34, 265 (1973).

[15] W. van Rijswijk, F. G. van den Berg, H. E. Keus, and W. J. Huiskamp, Physica B 113, 127 (1982).

[16] J. Crangle and G. M. Goodman, Proc. R. Soc. London A 321, 477 (1971).

[17] I. Alfter, E. Bodenstedt, B. Hamer, W. Knichel, R. Müßeler, R. Sajok, T. Schaefer, J. Schüth, and R. Vianden, Z. Phys. 347, 1 (1993). 